\title{
The sexual dysfunction in females with type 1 diabetes: Still an underestimated issue?
}

\author{
Djordje S Popovic $^{1 *}$, Ana Majic ${ }^{2}$ and Jovana Prodanovic-Simeunovic ${ }^{1}$ \\ ${ }^{1}$ Clinic for Endocrinology, Diabetes and Metabolic Disorders, Clinical Centre of Vojvodina, Medical Faculty, Novi Sad, Serbia \\ ${ }^{2}$ Department of Endocrinology, Diabetes and Clinical Pharmacology, Dubrava University Hospital, Zagreb, Croatia
}

\begin{abstract}
Sexuality is a significant part of female life and is under the impact of various life events, factors related to reproduction, relationships and socio-cultural specifics, but is also affected by different chronic medical conditions and related treatments. Physiologically, women`s sexual functioning consists of desire, arousal, plateau, orgasm and resolution. Female sexual dysfunction (FSD) is a highly prevalent, age-related and progressive problem. It has multiple causes and dimensions including biological, psychological and interpersonal elements. Several disorders, encompassing issues with sexual desire and arousal, orgasm malfunctioning and sexual pain, can be differentiated within the FSD entity. Diabetes is considered to be a major cause of the impaired sexual function. Although women with diabetes have even frequency of cardiovascular and neurological complications as males, FSD has been paid rather less attention in research than the sexual dysfunction in men with diabetes, particularly exclusive in females with type 1 diabetes mellitus (T1DM). Usually, FSD is more related to the psychosocial issues, especially depression, than to cardiovascular, metabolic and other traditional risk factors, which are more characteristic for men. The sexual difficulties among T1DM women require much more attention. Their regular annual in-depth evaluation should routinely include queries regarding the presence of depressive symptoms and their sexual functioning.
\end{abstract}

\section{Introduction}

Sexuality is a significant part of female life and it greatly influences its quality. It includes sexual identity, sexual function and sexual relationships [1]. During women's life sexuality is impacted by various life events, factors related to reproduction, relationships and sociocultural specifics, but is also grossly affected by different health issues, especially diverse chronic medical conditions and related treatments [2].

Physiologically, women's sexual functioning consists of desire, arousal, plateau, orgasm and resolution. It demands an adequate response to erotic stimuli by both automatic and sensory nervous system $[3,4]$. The normal sexual function requires various neuromuscular actions and ability of proper congestion of blood vessels, thus enabling boost in passion and excitation through an increment in diameter and length of clitoris, as well as by lubrication and wall enlargement of vagina $[3,4]$. The relaxation of smooth muscles of female genital erectile tissue and blood supply rise are dependent on dopamine, melanocortin, oxytocin, vasopressin and norepinephrine actions, which harmonize pathways responsible for processing and responding to sexual stimulation, whilst sexual desire is under the influence of different areas of brain $[3,4]$. Finally, estradiol and testosterone also increase stimulation and desire through the facilitation of dopamine release and the enhancement of nitric oxide synthesis, thus regulating the supply of blood and erectility of clitoris $[3,4]$.

Female sexual dysfunction (FSD) is a common, age-related and progressive, but often underestimated problem $[5,6]$. It has multiple causes and dimensions including biological, psychological and interpersonal elements [7]. Several disorders, encompassing issues with sexual desire and arousal, orgasm malfunctioning and sexual pain, can be differentiated within the FSD entity [8]. Disorders of sexual desire are the result of disturbance of either physical drive or motivation. Two major malfunctions can influence the female sexual desire: hypoactive sexual desire disorder, which is the most prevalent FSD, and sexual aversion disorder [8]. On the other hand, sexual arousal disorders are the result of inability of attaining or maintaining sufficient sexual excitation, whether persistently or recurrently, caused by interplay of different psychophysiological disarrangements [2,8]. Furthermore, orgasmic disorders are characterized by constant or occasional difficulty to attain or to delay orgasm, following an appropriate level of stimulation and arousal [8]. Finally, sexual pain disorders include dyspareunia (genital pain associated with coitus), which can appear due to local causes (vestibulitis, vaginal atrophy or vaginal infection), but can also be both physiologically and psychologically conditioned, and vaginismus (persistent or recurrent involuntary vagina`s outer third muscle spasm that hinders the normal vaginal penetration) $[8,9]$. All these disorders can cause personal distress and dissatisfaction due to the lack of feelings of relief and pleasure, expected from a sexual experience.

Diabetes mellitus is considered to be a major cause of the impaired sexual function. Males with both type 1 mellitus (T1DM) and type 2 diabetes mellitus (T2DM) have an increased risk of erectile dysfunction [10-12]. The severity and duration of diabetes and its cardiovascular and

${ }^{\star}$ Correspondence to: Djordje S POPOVIC, Clinic for Endocrinology, Diabetes and Metabolic Disorders, Clinical Center of Vojvodina Medical Faculty, University of Novi Sad, Hajduk Veljkova 1, 21000 Novi Sad, Serbia, Tel: +38163551606, +381214843758, Fax: +38121525081, E-mail: pitstop021@gmail. com; djordje.popovic@mf.uns.ac.rs; djordje.popovic@kcv.rs

Key words: depression, diabetes mellitus, female sexual dysfunction, sexuality, type 1 diabetes mellitus

Received: January 28, 2019; Accepted: February 15, 2019; Published: February 26,2019 
neurological complications, as well as the presence of comorbidities, higher age, antihypertensive treatment, higher body mass index (BMI) and tobacco consumption, are all triggering mechanisms responsible for an impairment in the sexual functioning [10-12]. Although women with diabetes have even frequency of cardiovascular and neurological complications as males, FSD has been paid rather less attention in research, particularly exclusive in females with T1DM. Also, the results of conducted studies are much less conclusive than of those carried out in men with diabetes [13]. The reasons for this include the absence of a unified FSD definition, deficiency of well-validated scales and sociological taboos associated with female sexuality [14].

An atherosclerotic process, nervous system impairment and endothelial dysfunction, often present in individuals with diabetes, all contribute to the FSD development [4]. Reduced arterial blood supply of the female pelvic anatomy, as a result of the atherosclerosis, inhibits the sexual response to stimuli through a decrease in enlargement of the vagina and the clitoris, vaginal lubrication and sexual stimulation, while chronic hyperglycemia causes a decrease in the mucus production and lubrication, and leads to higher incidence of bacterial and fungal infections [4]. Hormonal imbalances, which often accompany diabetes, can also induce a lapse of sexual desire, inadequate lubrication of the vagina, orgasm disturbances and coital pain [4]. Beside somatic causes, FSD in women with diabetes can be caused by various psychosocial factors. Poor body self-perception, fear of hypoglycemia, feeling of guilt, disgrace and discontentment associated with the presence of diabetes all badly affect the sexual functioning [4]. Finally, depression which frequently accompanies diabetes is associated with the lack of energy, absence of sexual desire and insufficiency of self-esteem, pleasure perception difficulties and psychosocial problems causing irritability and making obstacles in the relationship maintenance $[4,15]$.

The impaired sexual function among women with T1DM is a widespread issue that deserves much more attention than it has been attributed to so far. The aim of this review article is to briefly summarize the conclusions of the studies conducted to date on this important and sensitive topic.

\section{Female sexual dysfunction and type 1 diabetes mellitus}

As it was mentioned before, earlier studies analysing FSD in diabetes are seldom, often biased by substantial methodological weaknesses, like small sample sizes and the absence of appropriate diabetes characterization, particularly regarding glycemic control, cardiovascular and neurological complications, psychological issues related to diabetes and existence of depression $[4,13]$. However, the results of studies investigating FSD among individuals with T1DM often point out different culprits for this major disorder, in comparison to the male population suffering from T1DM.

Ezlin et al. studied 120 females with T1DM and 180 age-matched control subjects in order to investigate the prevalence of sexual issues in females with T1DM, to compare this prevalence rate with the one of healthy females and to study the impact of somatic factors associated with diabetes on female sexuality, as well as the nature of a collision of different psychological points on the sexual functioning of both T1DM and healthy women [16]. The authors found that females with T1DM reported the sexual dysfunction much more frequently than healthy women (27 vs. $15 \%$ ), with a significant distinction found only for a decreased lubrication [16]. Also, there was no association between FSD and age, BMI, diabetes duration, glycated hemoglobin (HbAlc), medication usage, menopausal status and the presence of chronic complications of diabetes, although women with developed diabetic complications had notably more sexual dysfunctions [16]. Finally, both T1DM and healthy females with FSD pointed out lower overall quality of the marital relationship and more symptoms of depression than women with no difficulties in the sexual functioning, with a depression as a significant predictor for FSD in both groups [16]. In their other study, the same authors analyzed the prevalence of sexual dysfunction aiming to depict how expositive and psychological variables, complications of diabetes and the sexual dysfunction interact in diabetes and which are the predictors of sexual dysfunction in 240 adult T1DM individuals [17]. In this study, the sexual dysfunction was reported by $27 \%$ of females and $22 \%$ of males, with no differences in type of the outlined sexual dysfunction among genders [17]. Unlike in men, the sexual dysfunction was not related to age, BMI, diabetes duration and the presence of complications of diabetes among women, while on the other hand, in females, but not in males, the sexual dysfunction was associated with depression and with the quality of relationship with the sexual partner [17]. Older age and the presence of complications in men, and depression in women, were found to be the significant predictors of the sexual dysfunction [17]. In their more recent study, the same group investigated the frequency and risk factors related with the sexual dysfunction in a cohort of women with T1DM from the Diabetes Control and Complications Trial/Epidemiology of Diabetes Interventions and Complications (DCCT/EDIC) study [18]. The authors reported that $35 \%$ of women met the criteria for FSD, with a loss of libido, orgasm disorders, impairments in lubrication and arousal, and sexual pain as the most frequent complaints [18]. The sexual dysfunction was positively associated with age, matrimonial and menopausal status, the presence of microvascular complications of diabetes and depression, but in a multivariate analysis, only depression and matrimonial status were significant predictors of FSD [18]. Dimitropoulos et al. analyzed the sexual function and distress, as well as the general health status in 44 premenopausal females with uncomplicated T1DM and 47 healthy women [19]. In this research, FSD was present with higher frequency in T1DM women (15.9\%), in comparison to the controls (2.1\%), with desire, arousal and satisfaction as domains of the sexual functioning notably altered in the T1DM group [19]. The sexual dysfunction was associated with anxiety, depression and a lower educational level, while factors linked to diabetes were not related to FSD [19]. Bak et al. evaluated sexuality in women and men with T1DM and found that diabetes causes sexual disorders in one third of women and in one half of men, with higher frequency in persons with diabetic complications and in those with the HbAlc level greater than $6.5 \%$ [20]. Maiorino et al. analyzed the prevalence and risk factors associated with FSD in young females with T1DM treated with diverse regimens of the intensive insulin therapy [21]. Interestingly, they found that the T1DM women wearing an insulin pump showed FSD prevalence similar to the one of healthy females, but that sexual functioning was remarkably altered in T1DM females on multiple daily injections therapy, with depression and the mental health condition as independent predictors for the sexual dysfunction in T1DM females [21]. On the other hand, Mazzilli et al. investigated the differences between sexuality in the context of presence of T1DM and T2DM and enrolled 49 T1DM women, 24 T2DM women and 45 healthy controls [22]. Authors found greater prevalence of FSD in T1DM women (51\%), but not in T2DM women (17\%), in comparison to the healthy individuals (9\%) [22]. Issues with arousal, lubrication, orgasm and dyspareunia were more frequent in T1DM women, while only desire was reduced in both T1DM and T2DM women, comparing to the controls [22].

Rare studies examined the pathophysiological mechanisms potentially involved in FSD development in T1DM women. Both et 
al. analyzed the differences in vaginal blood flow, subjective sexual response and clitoral sensitivity between females with T1DM and control subjects, as well as their association with the presence of diabetic complications [23]. None of the parameters were significantly different between T1DM and healthy women, although the T1DM women with retinopathy showed lower vaginal pulse amplitude, while the T1DM women with neuropathy showed higher sensation thresholds for the vibrotactile stimulation of clitoris [23]. Hotaling et al. evaluated coherence between cardiovascular autonomic neuropathy, FSD and urinary incontinence among 580 women with T1DM in the DCCT/EDIC study [24]. Authors reported that in the long-standing T1DM, cardiovascular autonomic neuropathy may predict the FSD development [24].

\section{Diagnostic and treatment approaches}

The diagnostic and treatment approaches should be very careful and tactful. Studies have reported that women are willing to talk about their sexual problems but prefer to discuss them with health practitioners who are well trained to successfully initiate the conversation and have sufficient knowledge to identify the sexual issues of interest [3]. A woman and her partner can be referred to an experienced sexual relationship therapist, who uses techniques such as the Cognitive Behavioural Therapy and Sensate Focusing, helping couples to learn sensual tactile techniques, in order to try to decrease possible tension and improve performance, thus helping them to overcome the existing sexual problems [25]. These psychological approaches can be helpful, although cultural and religious preferences should be taken into account when suggesting them [26]. Other therapeutic options include the usage of vaginal lubricants, clitoral engorgement devices and various potentially useful medications (flibanserin, testosterone, bupropion, sildenafil etc.) $[3,27,28]$. The normal sexual functioning is very important for women with diabetes, especially for young T1DM individuals, and this issue certainly needs to be addressed during the adequate pre-marriage counselling [29].

\section{Conclusion}

The sexual dysfunction is a common impairment in females with T1DM, which has a negative impact on the quality of life and relations with a sexual partner. In contrast to males, the sexual dysfunction in females is usually much more related to the psychosocial issues, especially depression, than to cardiovascular, metabolic and other traditional risk factors. The sexual difficulties among T1DM women require much more attention, both scientifically and practically. Their regular annual in-depth evaluation should routinely include queries regarding the presence of depressive symptoms and their sexual functioning. The size and importance of this clinical problem requires additional research in the future.

\section{Authors disclosures}

None.

\section{Funding}

This research did not receive any specific grant from funding agencies in the public, commercial, or not-for-profit sectors.

\section{References}

1. Association of Reproductive Health Professionals (2005) Women's sexual health in midlife and beyond. Washington, DC: Association of Reproductive Health Professionals.

2. Amato P (2006) Categories of female sexual dysfunction. Obstet Gynecol Clin North Am 33: 527-534. [Crossref]
3. Rochester-Eyeguokan C, Meade L (2017) A practical approach to managing hypoactive sexual desire disorder in women with diabetes. Diabetes Ther 8: 991-998.

4. Clayton AH (2010) The pathophysiology of hypoactive sexual desire disorder in women. Int J Gynaecol Obstet 110: 7-11.

5. Raina R, Pahlajani G, Khan S, Gupta S, Agarwal A, et al. (2007) Female sexual dysfunction: classification, pathophysiology, and management. Fertil Steril 88: 12711284.

6. Laumann EO, Paik A, Rosen RC (1999) Sexual dysfunction in the United States prevalence and predictors. JAMA 281: 537-544. [Crossref]

7. Basson R, Leiblum S, Brotto L, Derogatis L, Fourcroy J, et al. (2003) Definitions of women's sexual dysfunctions reconsidered: advocating expansion and revision. $J$ Psychosom Obstet Gynaecol 24: 221-229.

8. Mimoun S, Wylie K (2009) Female sexual dysfunctions: definitions and classification Maturitas 63: 116-118. [Crossref]

9. Berman JR, Berman LA, Kanaly KA (2003) Female sexual dysfunction: new perspectives on anatomy, physiology, evaluation and treatment. EAU Update Series 1: 166-177.

10. Brown JS, Wessells H, Chancellor MB, Howards SS, Stamm WE, et al. (2005) Urologic complications of diabetes. Diabetes Care 28: 177-185. [Crossref]

11. Fedele D, Bortolotti A, Coscelli C, Santeusanio F, Chatenoud L, et al. (2000) Erectile dysfunction in type 1 and type 2 diabetics in Italy. On behalf of Gruppo Italiano Studio Deficit Erettile nei Diabetici. Int J Epidemiol 29: 524-531.

12. Rhoden EL, Ribeiro EP, Riedner CE, Teloken C, Souto CA (2005) Glycosylated haemoglobin levels and the severity of erectile function in diabetic men. BJU Int 95 : 615-617. [Crossref]

13. Enzlin P, Mathieu C, Vanderschueren D, Demyttenaere K (1998) Diabetes mellitus and female sexuality: a review of 25 years' research. Diabet Med 15: 809-815.

14. Althof SE, Rosen RC, DeRogatis L, Corty E, Quirk F, et al. (2005) Outcome measurement in female sexual dysfunction clinical trials: review and recommendations. J Sex Marital Ther 31: 153-166.

15. Palacios S, Castaño R, Grazziotin A (2009) Epidemiology of female sexual dysfunction Maturitas 63: 119-123. [Crossref]

16. Enzlin P, Mathieu C, Van den Bruel A, Bosteels J, Vanderschueren D, et al. (2002) Sexual dysfunction in women with type 1 diabetes: a controlled study. Diabetes Care 25: 672-677. [Crossref]

17. Enzlin P, Mathieu C, Van Den Bruel A, Vanderschueren D, Demyttenaere K (2003) Prevalence and predictors of sexual dysfunction in patients with type 1 diabetes. Diabetes Care 26: 409-414.

18. Enzlin P, Rosen R, Wiegel M, Brown J, Wessells H, et al. (2009) Sexual dysfunction in women with type 1 diabetes. Diabetes Care 32: 780-785.

19. Dimitropoulos K, Bargiota A, Mouzas O, Melekos M, Tzortzis V, et al. (2012) Sexual functioning and distress among premenopausal women with uncomplicated type 1 diabetes. J Sex Med 9: 1374-1381.

20. Bak E, Marcisz C, Krzeminska S, Dobrzyn-Matusiak D, Foltyn A, et al. (2018) Does type 1 diabetes modify sexuality and mood of women and men? Int $J$ Environ Res Public Health 15: pii:E958. [Crossref]

21. Maiorino MI, Bellastella G, Castaldo F, Petrizzo M, Giugliano D, et al. (2017) Sexual function in young women with type 1 diabetes: the METRO study. $J$ Endocrinol Invest 40: 169-177. [Crossref]

22. Mazzilli R, Imbrogno N, Elia J, Delfino M, Bitterman O, et al. (2015) Sexual dysfunction in diabetic women: prevalence and differences in type 1 and type 2 diabetes mellitus. Diabetes Metab Syndr Obes 8: 97-101.

23. Both S, Ter Kuile M, Enzlin P, Dekkers O, van Dijk M, et al. (2015) Sexual response in women with type 1 diabetes mellitus: a controlled laboratory study measuring vaginal blood flow and subjective sexual arousal. Arch Sex Behav 44: 1573-1587.

24. Hotaling JM, Sarma AV, Patel DP, Braffett BH, Cleary PA, et al. (2016) Cardiovascular autonomic neuropathy, sexual dysfunction, and urinary incontinence in women with type 1 diabetes. Diabetes Care 39: 1587-1593.

25. Viigimaa M, Vlachopoulos C, Doumas M (2015) Erectile dysfunction in hypertension and cardiovascular disease: a guide for clinicians. Basel: Springer International Publishing Switzerland. 
26. Phillips A, Phillips S (2015) Recognising female sexual dysfunction as an essential aspect of effective diabetes care. Appl Nurs Res 28: 235-238.

27. Billups KL (2002) The role of mechanical devices in treating female sexual dysfunction and enhancing the female sexual response. World J Urol 20: 137-141. [Crossref]
28. Lo Monte G, Graziano A, Piva I, Marci R (2014) Women taking the "blue pill" (sildenafil citrate): such a big deal? Drug Des Devel Ther 8: 2251-2254. [Crossref]

29. Priya G, Kalra B, Grewal E, Dardi IK (2018) Premarriage counselling in type 1 Diabetes. Indian J Endocrinol Metab 22: 126-131. [Crossref]

Copyright: (C2019 Popovic DS. This is an open-access article distributed under the terms of the Creative Commons Attribution License, which permits unrestricted use, distribution, and reproduction in any medium, provided the original author and source are credited. 